\title{
Caracterización de ficocianina por espectrofotometría de masas
}

\section{Characterization of phycocyanins by mass spectrophotometry}

\author{
L. B. González- Fosados (iDa , B. K. González-Chávez (D)a ,A. Garrido-Hernández iDa , G. Carrillo-Sancen iDa, G. I. Cerón \\ Montes iDa, F. J. Martínez-Valdez iDa \\ a Universidad Tecnológica de Tecámac, 55740, Tecámac, Edo. Méx, México.
}

\begin{abstract}
Resumen
La espectrometría de masas (EM) es uno de los métodos analíticos más utilizados por su sensibilidad, límites de detección, rapidez y en combinación con la cromatografía obtiene datos respecto a estructura y composición de proteínas. Aplicaciones biotecnológicas de complejos proteicos demandan dicha información. Por medio de la EM se pueden obtener estos datos, con alta reproducibilidad y una muestra de $0.1 \mathrm{a} 1 \mu \mathrm{g}$. Se ha reportado que la ficocianina presenta un alto poder antioxidante, antiinflamatorio, anticancerígeno e incluso ha demostrado potencial antiviral y posibles usos en trastornos relacionados con el COVID-19. A pesar de su utilidad, se desconoce en gran medida su estructura e interacciones. Por lo que, se realizó una revisión bibliográfica del análisis por EM donde se detectaron las subunidades características de la ficocianina ( $\alpha$ y $\beta$ ) con pesos moleculares de $17 \mathrm{kDa}$ y $18-21 \mathrm{kDa}$, además, obteniendo la secuenciación con MASCOT, NIST Mass and Fragment Calculator, entre otros softwares.
\end{abstract}

Palabras Clave:

Cianobacteria, Ficocianina, Caracterización, Espectrofotometría de masas, Secuenciación de proteína.

\begin{abstract}
Mass spectrometry (MS) is one of the most widely used analytical methods due to it's sensitivity, detection limits, speed, and in combination with chromatography, obtaining data regarding protein structure and composition. Biotechnological applications of protein complexes require such information. By means of MS, these data can be obtained, with high reproducibility and a sample size of 0.1 to $1 \mu \mathrm{g}$. It has been reported that phycocyanin has high antioxidant, anti-inflammatory, and anticancer properties and has even demonstrated antiviral potential and possible uses in COVID-19 related disorders. Despite their usefulness, their structure and interactions are largely unknown. Therefore, a literature review of MS analysis was performed where the characteristic phycocyanin subunits $(\alpha$ and $\beta$ ) with molecular weights of $17 \mathrm{kDa}$ and $18-21 \mathrm{kDa}$ were detected, in addition to obtaining sequencing with MASCOT, NIST Mass and Fragment Calculator, among other software.
\end{abstract}

Keywords:

Cyanobacteria, Phycocyanin, Characterization, Mass spectrometry, Protein sequencing.

\section{Introducción}

La ficocianina forma parte de las ficobiliproteínas y es el pigmento fotosintético más abundante en la Spirulina, siendo el principal componente que proporciona la actividad antioxidante de esta cianobacteria. Este metabolito genera efectos biológicos beneficiosos para el ser humano, con un alto poder antioxidante, antiinflamatorio y anticancerígeno (Hsieh-Lo et al., 2019).

Incluso se ha demostrado como un potencial antiviral y su posible utilidad en el tratamiento de trastornos relacionados con el reciente brote de COVID-19 causado por el SARS-CoV2, esto último es posible gracias a sus propiedades nutracéuticas, antiinflamatorias, inmunoestimuladoras e inmunomoduladoras, siendo un compuesto de salud preventiva que requiere más investigación (Ratha et al., 2020).

Además, los efectos beneficiosos, como la actividad antioxidante, sólo se proporcionan cuando la ingesta de compuestos bioactivos es regular y, por lo tanto, una variedad de formas para suministrar productos que contengan estos compuestos también es vital para permitir su consumo frecuente (Rivera et al., 2021).

\footnotetext{
*Autor para la correspondencia: berenicegonzalezfosados@gmail.com

Correo electrónico: berenicegonzalezfosados@gmail.com (Lluvia Berenice González-Fosados), karina.chavez100416@gmail.com (Brenda K. González-Chavez), agarridoh@uttecamac.edu.mx (Aristeo Garrido-Hernández), GcarrilloS@uttecamac.edu.mx (Gabriela Carrillo-Sancen), gceronm@uttecamac.edu.mx (Genaro I. Cerón-Montes), fco.jav.mv@gmail.com (Francisco J. Martínez-Valdez).
} 
Las mayores concentraciones y pureza de este pigmento están directamente relacionadas con la manipulación de la biomasa en el laboratorio y/o la industria, ya que la estabilidad de la biomolécula depende del $\mathrm{pH}$, la temperatura, la exposición a la luz, el tiempo de extracción, el tipo de disolvente y la relación biomasa-disolvente (Fratelli et al., 2021). Por lo tanto, es de gran importancia la caracterización de la ficocianina debido al uso de esta ficobiliproteína como pigmento azul en alimentos procesados o incluso para aplicaciones biomédicas (Raj et al., 2020).

Atendiendo lo anterior, la espectrofotometría de masas es una técnica que se destaca entre los métodos analíticos existentes, debido a sus extraordinarias características, como límites de detección, rapidez o la posibilidad de potenciar su función combinándola con cromatografía. Por lo que, se propone un estudio de metodologías de espectrofotometría de masas para la caracterización de ficocianina.

\section{Ficocianina}

Para este apartado se consideró a la cianobacteria Spirulina (Arthrospira) como fuente principal de la ficocianina, se realiza una descripción de la ficocianina y su caracterización por medio de la técnica espectrofotometría de masas.

\subsection{Spirulina}

Las cianobacterias del género Spirulina han sido uno de los pocos microorganismos fotosintéticos que se han producido a gran escala y comercializado en todo el mundo como alimento y fuente de varios productos de alto valor añadido (Olguín et al., 2019).

Se trata de una microalga simbiótica, multicelular y filamentosa de color verde azulado con gran capacidad de fijar el nitrógeno del aire. Se reconoce por la disposición de los tricomas cilíndricos multicelulares en una hélice abierta a la izquierda en toda su longitud. La superficie del cuerpo de la Spirulina es lisa y sin recubrimiento, por lo que puede ser fácilmente digerida a través de sistemas enzimáticos simples y el principal pigmento fotosintético es la ficocianina, de color azul (Ismaiel et al., 2016).

La Spirulina se ha utilizado durante más de 1.000 años como fuente de alimentos, por tener un elevado contenido de proteínas del 55\% a 70\% del peso seco total (Prabakaran et al., 2020).

\subsection{Ficocianina}

La ficocianina de la Spirulina, es una proteína que tiene un color azul brillante, se compone de dos subunidades y se vende como colorante para productos alimenticios y cosméticos (Chen et al., 2014).

Hasta un $20 \%$ de ficocianina está presente en la fracción proteica de la Spirulina. Existe como monómeros, trímeros o hexámeros y también como oligómeros en pequeñas cantidades. Las ficocianinas incluyen tanto la C-ficocianina como la aloficocianina (Grover et al., 2021).

Las ficocianinas son abundantes pigmentos captadores de luz que desempeñan un importante papel en la cascada de transferencia de energía de los fotosistemas de las cianobacterias procariotas y las algas rojas eucariotas. Estas proteínas tienen importantes aplicaciones biotecnológicas, ya que pueden utilizarse en la industria nutracéutica, farmacéutica y en la investigación biomédica (Ferraro et al., 2020).

Esta ficobiliproteína se ha asociado con beneficios para la salud debido a sus propiedades antioxidantes y antiinflamatorias. El potencial anticancerígeno de la ficocianina se ha informado en diferentes líneas de células cancerosas de hígado, colon, pulmón y mama, entre otros (Koníčková et al., 2014). Los antioxidantes pueden prevenir la producción de especies reactivas de oxígeno
(ROS), que están relacionadas con diversas enfermedades como cáncer, diabetes mellitus, enfermedades inflamatorias, así como neuroenfermedades degenerativas (Hsieh-Lo et al., 2019).

\subsection{Espectrofotometría de masas}

Las características de la espectrometría de masas la han elevado a una posición destacada entre los métodos analíticos: por su sensibilidad, límites de detección, rapidez, diversidad de sus aplicaciones y la posibilidad de combinarla con la cromatografía para un mejor análisis de secuenciación de proteínas. La capacidad de la espectrometría de masas para identificar un compuesto en una muestra se maximiza cuando la muestra es pura. La combinación de espectrometría de masas con un sistema de entrada cromatográfica se ha convertido en un pilar del análisis instrumental (Busch, 2003).

Esto último es importante, ya que los cromatógrafos de gases o líquidos, como dispositivos de entrada a los espectrómetros de masas, separan mezclas complejas de compuestos químicos para su posterior detección y reconocimiento, la selectividad múltiple de las técnicas combinadas se muestra en la determinación cualitativa de diferentes compuestos orgánicos en mezclas y matrices (Milman, 2015)

En la química analítica, las aplicaciones más recientes se orientan sobre todo a los problemas bioquímicos, como el proteoma, el metaboloma, con elevado rendimiento en el descubrimiento de fármacos y el metabolismo. Otros usos analíticos se aplican de forma rutinaria en el control de la contaminación, el control de los alimentos, la ciencia forense, los productos naturales o la supervisión de procesos. Van Bergen et al., (2021), mencionan su utilidad, como en la física atómica, la física de las reacciones, la cinética de las reacciones, la geocronología, análisis químico inorgánico, reacciones de moléculas iónicas, determinación de parámetros termodinámicos.

La espectrometría de masas ha progresado durante la década entre 1995 y 2005. Este progreso llevó a la aparición de instrumentos totalmente nuevos. Se desarrollaron nuevas fuentes de presión atmosférica, se perfeccionaron los analizadores existentes y nuevos instrumentos híbridos mediante nuevas combinaciones de analizadores. Se propuso un analizador de masas que utiliza la separación de iones en un campo eléctrico oscilante llamado orbitrap. Esto ha permitido desarrollar nuevas aplicaciones. Por citar algunos ejemplos, se obtuvieron los primeros espectros de un virus intacto y de complejos no covalentes. Se desarrolló una nueva espectrometría de masas de alto rendimiento desarrollada para satisfacer las necesidades de la proteómica, la metabolómica y otras "ómicas" (Hoffmann et al., 2007).

Por lo cual, es necesario conocer la técnica de caracterización por espectrofotometría de masas para definir las propiedades de la ficocianina a estudiar, permitiendo posteriormente describir los factores óptimos que debe comprender este elemento durante su extracción y purificación, así mismo, para validar el comportamiento o aplicaciones de la proteína. En la siguiente sección se presentan los procesos de técnicas propuestas en diversos artículos, para la caracterización de ficocianina por espectrometría de masas (Fig. 1), así también aspectos importantes como la purificación y preparación de la muestra para su análisis. 


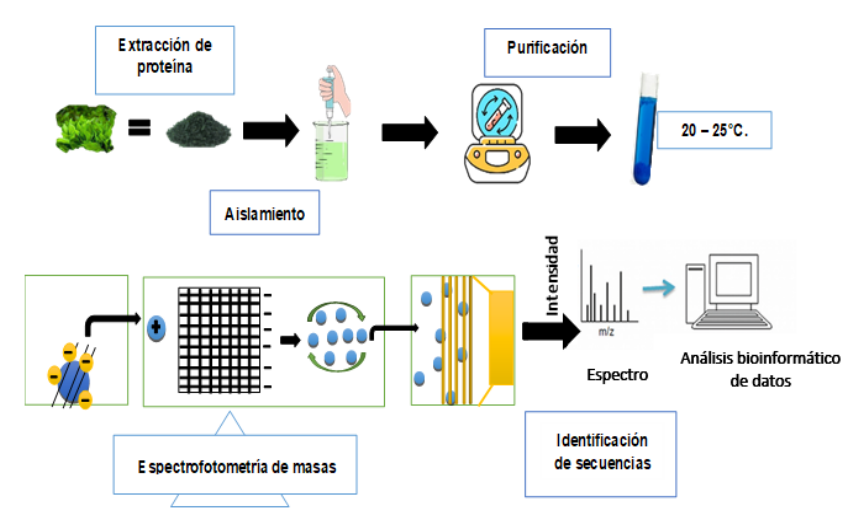

Figura 1. Esquema general de la metodología para espectrofotometría de masas.

\section{Extracción de Ficocianina}

\subsubsection{C-ficocianina (Galdieria phlegrea)}

Ferraro et al. (2020), mencionan que la proteína fue extraída a partir de G. phlegrea (cepa 009) la cual provino de Algal Collection University Federico II (ACUF). Para iniciar con el proceso de la investigación, explican que el aislamiento de la $\mathrm{C}$ ficocianina a partir de $G$. phlegrea (CFGp) se llevó a cabo por ultrafiltración. El extracto de proteína total obtenido por procedimientos convencionales de alta presión fue purificado mediante el uso de una membrana con peso molecular de $100 \mathrm{kDa}$ (se descartó el filtrado y se recogió el permeado), el proceso se llevó a cabo a temperatura ambiente y se evaluó el grado de pureza de CFGp primero midiendo la relación A620nm /A280nm, luego por SDS-PAGE nativa.

\subsubsection{C-Ficocianina (Geitlerinema sp)}

Patel, Rastogi, Trivedi y Madamwar (2018) indicaron que su principal precursor fue la cianobacteria $G$. sp, se aisló de la costa de Bet-Dwarka, Gujarat, India. Los cultivos se realizaron en un medio de crecimiento estéril ASN III a $28 \pm 2{ }^{\circ} \mathrm{C}$ bajo un ciclo de iluminación de 12/12 h de luz/oscuridad. El cultivo aislado se identificó mediante un examen microscópico utilizando un microscopio de luz (MPN-400, Carl-Zeiss, Alemania), un microscopio de fluorescencia (BX-41, Olympus, Japón) y un microscopio electrónico de barrido (Nova NanoSEM 450, FEI Ltd., EE.UU.). Posteriormente, la biomasa celular de los cultivos exponenciales de $G$. sp se recogió centrifugando la suspensión celular a 12, $000 \times \mathrm{g}$ durante 10 minutos. La biomasa se resuspendió en tampón de extracción (fosfato de potasio $20 \mathrm{mM}$ : $\mathrm{KPB}, \mathrm{pH} 7$ ) y se utilizó para la extracción de proteínas mediante una sonicación suave a $20 \mathrm{kHz} \pm 50 \mathrm{~Hz}$ durante $1-2$ min y ciclos repetidos de congelación $\left(-80^{\circ} \mathrm{C}\right)$ y descongelación $\left(20^{\circ} \mathrm{C}\right)$ para lisar las células. El lisado se centrifugó a 14, 000×g durante 20 minutos a $4{ }^{\circ} \mathrm{C}$ para eliminar la clorofila y otros restos celulares. La suspensión libre de células de ficocianina (FC) se enriqueció por precipitación con sulfato de amonio de 0 a $25 \%(\mathrm{p} / \mathrm{v})$ con agitación continua durante 1-2 h. Los homogeneizados se centrifugaron $(14,000 \times \mathrm{g}, 20 \mathrm{~min})$ y se recuperaron los sobrenadantes de color azul cobre, se precipitaron añadiendo sulfato de amonio hasta el $45 \%(\mathrm{p} / \mathrm{v})$ y se incubaron durante la noche a $4{ }^{\circ} \mathrm{C}$. Las proteínas precipitadas se precipitaron por centrifugación (centrifugadas a $12,000 \times g, 15 \mathrm{~min}$ ) y se disolvieron en $20 \mathrm{mM}$ de KPB. La suspensión de proteínas se dializó (Sigma, $12 \mathrm{kDa}$ ) contra el tampón de muestra durante una noche a $4{ }^{\circ} \mathrm{C}$.

\subsubsection{Alga roja marina (Centroceras clavulatum)}

Nair, Krishna, Velayudhan y Panikkar (2018) expresan que la muestra de algas fue extraída de la región costera de Kollam, Kerala $\left(8^{\circ} 53^{\prime} \mathrm{N}, 76^{\circ} 33^{\prime} \mathrm{E}\right)$ y fue identificado como Centroceras clavulatum a través de sus características morfológicas. Posteriormente las muestras de algas fueron lavadas adecuadamente con agua destilada estéril y se almacenaron a -80 ${ }^{\circ} \mathrm{C}$ hasta su uso. Posteriormente se utilizaron $70 \mathrm{~g}$ de $C$. clavulatum, se homogeneizó en $200 \mathrm{~mL}$ de fosfato de sodio 0,1 M, con pH 7.4 y la mezcla se centrifugó a 20,000× $g$ durante 20 min. La temperatura se mantuvo a $4{ }^{\circ} \mathrm{C}$. El sobrenadante así obtenido se precipitó secuencialmente utilizando sulfato de amonio $\left(\left(\mathrm{NH}_{4}\right)_{2} \mathrm{SO}_{4}\right)$ al $30 \%, 40 \%$ y $50 \%$ y la proteína precipitada se recuperó por centrifugación. Los gránulos se disolvieron en un tampón de fosfato de sodio 0,05 M, pH 7,4. La fracción proteica obtenida tras el corte del $40 \%$ de $\left(\left(\mathrm{NH}_{4}\right)_{2} \mathrm{SO}_{4}\right)$ se sometió a diálisis con tampón de fosfato de sodio, $\mathrm{pH}$ 7,4 y la concentración de proteína la determinaron siguiendo el ensayo estándar de Bradford.

\subsubsection{C-Ficocianina (Microalga Spirulina)}

Minic et al (2016) obtuvieron la biliproteína purificando un extracto de proteína de polvo comercial de Spirulina (de Hawaiian Spirulina Pacifica, Nutrex, EE. UU,) de acuerdo con el protocolo descrito. Posteriormente la extracción de ficocianinas crudas se realizó usando tampón de fosfato de sodio $20 \mathrm{mM}, \mathrm{pH}$ 6.8; la suspensión de polvo-tampón se mezcló durante 3 horas a temperatura ambiente. La concentración de C- ficocianina (C-FC) se determinó mediante espectrofotometría UV-vis. Se aisló ficocianobilina (FCB) y la concentración se determinó mediante un coeficiente de absorción molar de $37900 \mathrm{M}^{-1} \mathrm{~cm}^{-1}$ a $680 \mathrm{~nm}$. La digestión con pepsina de $\mathrm{C}-\mathrm{FCb}$ se realizó en fluido gástrico simulado (FGS). Brevemente, se agregaron $80 \mu \mathrm{L}$ de C-FC ( $5 \mathrm{mg}$ / $\mathrm{mL}$ ) a $760 \mu \mathrm{L}$ de FGS ( $84 \mathrm{mM} \mathrm{HCl}$ y $35 \mathrm{mM} \mathrm{NaCl}, \mathrm{pH} 1.2)$, que contiene 1 unidad de pepsina (de mucosa gástrica porcina, $2546 \mathrm{U}$ / mg; Sigma-Aldrich, EE. UU.) por $\mu$ g de C-FC. La mezcla se incubó a $37^{\circ} \mathrm{C}$ y se tomaron alícuotas de $60 \mu \mathrm{L}$ a $0.5 \mathrm{~min}, 5 \mathrm{~min}$, 30 min, 1 h, 2 h, 5 h y 24 h después del inicio de la incubación. Cada alícuota fue apagada mediante la adición de $20 \mu \mathrm{L}$ de $\mathrm{NaHCO}_{3} 300 \mathrm{mM}, \mathrm{pH}$ 11. Se prepararon alícuotas a tiempo cero inactivando la pepsina que contenía FGS antes de añadir C-FC. Se realizó una electroforesis en gel de poliacrilamida SDS (SDSPAGE) de digestiones en condiciones reductoras y los geles se tiñeron usando Coomassie Brilliant Blue R-250. Para la identificación de la estructura de los cromopéptidos y sus evaluaciones de bioactividad, se prepararon mezclas de digestión a mayor escala y la digestión se realizó durante un período de incubación de $24 \mathrm{~h}$.

\subsection{Análisis de ficocianina por espectrofotometría de masas}

\subsubsection{Análisis cromatografía líquida-espectrometría de masas (CL-EM/EM)}

Ferraro et al (2020), describen que las muestras de péptidos se cargaron mediante un muestreador automático (Surveyor EM Pump Plus y Micro AS) en un Michrom C18 Captrap posteriormente se introdujeron directamente en un Orbitrap LTQVelos EM (Thermo Fisher Scientific, Surrey, Reino Unido) a 
través de una columna capilar C18 de sílice fundida (Nikkyo Technos CO, Tokio, Japón) y un ion nanoelectrospray fuente donde la fase móvil comprendía $\mathrm{H}_{2} \mathrm{O}$ con ácido fórmico al $0.1 \%$ (tampón A) y acetonitrilo al $100 \%$ con ácido fórmico al $0,1 \%$ (tampón B). Mencionan que el gradiente varió del 5\% al 30\% de tampón B en 95 min, seguido de un 30\% a 60\% B en 15 min y un gradiente escalonado a $85 \%$ B durante 5 min con un flujo de 0.42 $\mu \mathrm{L} \mathrm{min}{ }^{-1}$. Finalmente, el sistema vuelve a las condiciones iniciales de $5 \% \mathrm{~B}$.

A continuación, procedieron a la fragmentación EM/EM dependiente de los datos en modo centroide del ion más intenso del barrido de estudio, utilizando la disociación inducida por colisión (DIC) en la trampa de iones lineal: energía de colisión normalizada 35\%; activación $\mathrm{Q}=0,25$; voltaje de electropulverización $1,5 \mathrm{kV}$; temperatura capilar $200^{\circ} \mathrm{C}$; y ancho de aislamiento 2.00. Este evento de escaneo EM/EM se repitió para los 20 picos más importantes del barrido EM; los iones seleccionados se excluyeron dinámicamente durante 30s. Los iones con carga única se excluyeron del análisis EM/EM; para la adquisición de datos se utilizó el software Xcalibur versión 2.1.0 SP1 build 1160 (Thermo Fisher Scientific, Reino Unido).

\subsubsection{Análisis de espectrofotometría de masas (MALDI)}

Patel et al (2018), mencionan que a la mezcla de péptidos purificada se le añadió ácido $\alpha$-ciano-4-hidroxicinámico (5 $\mathrm{mg} / \mathrm{mL}$ ) en el buffer TA para preparar la solución matriz. Se secó en la placa objetivo MALDI para proceder al análisis. La huella de masa peptídica (HMP) de la CF se obtuvo en el modo de reflexión positiva mediante el espectrómetro de masas de ionización de desorción láser asistida por matriz (MALDIToF/ToF) (ULTRAFLEX III, Bruker Daltonics, USA). Los espectros se registraron en el rango de 0-4000 Da con una acumulación de mil disparos para cada muestra. Los espectros de masas de los péptidos seleccionados se generaron a una tensión de aceleración de 1 ke mediante disociación impulsada por colisión gas/aire a una presión de 10-6 Torr.

\subsubsection{Espectrometría de masas en tándem de cromatografía líquida (LC-EM/EM)}

Para el análisis de proteínas Nair et al (2018), utilizan una cromatografía HPLC de flujo nano Agilent serie 1260 junto con una serie 6540. Se utilizó UHD Q-TOF (Agilent Technologies) y una interfase HPLC Chip Cube. Las muestras de proteína digeridas se infundieron a través de un chip de fase inversa

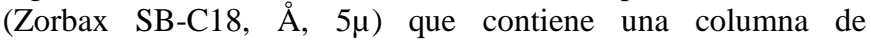
enriquecimiento de $40 \mathrm{~nL}$ y una columna analítica de $75 \mathrm{~mm}$ x 43 $\mathrm{mm}$. Agua que contiene ácido fórmico al (A) $0,1 \%$ y (B) acetonitrilo al $90 \%$ en agua más ácido fórmico al $0,1 \%$ se utilizaron como fase móvil. El caudal mantenido para la columna de enriquecimiento y separación fue de $3 \mu \mathrm{L} / \min$ y $300 \mathrm{~nL} / \min$, respectivamente. La nanobomba se hizo funcionar con el siguiente gradiente: 0 min, $2 \%$ de B; 3 min, $2 \%$ de B; 35 min, $45 \%$ de B; 40 min, $90 \%$ B. Todos los datos de EM se adquirieron en ionización positiva y modo centroide utilizando el software de adquisición de datos Mass Hunter de Agilent (versión B.05.01). El voltaje capilar se mantuvo en $1700 \mathrm{~V}$ y el voltaje del fragmentador se estableció en $175 \mathrm{~V}$. El flujo de gas seco y la temperatura del gas seco se mantuvieron a $6 \mathrm{~L} / \min$ y $320^{\circ} \mathrm{C}$, respectivamente. Los siguientes fueron los otros parámetros importantes de adquisición de datos: rango de escaneo EM, 300-3000 $\mathrm{m} / \mathrm{z}$; rango de escaneo automático EM / EM, 50-2000 m/z; velocidad de escaneo, 3 espectros/seg; selección de precursores, máximo de 5 precursores; cargos preferidos por tríptico digiere, 2, 3,> 3; cargas preferidas para la digestión de quimotripsina y proteasa V8, 1, 2, 3,> 3; exclusión activa, después de 3 espectros durante 0,5 min. Los datos adquiridos se analizaron utilizando el Software de análisis cuantitativo Agilent Mass Hunter, versión B.07.00.

Los datos adquiridos de EM se convirtieron a un archivo de formato genérico de mascot (.mgf) utilizando Mass Hunter software y se buscó en una base de datos de proteínas de algas creada internamente utilizando Mascot (versión 2.5). Para las búsquedas de Mascot, las cisteínas carbamidometiladas se establecieron como modificaciones fijas, la oxidación de la metionina y la desaminación se establecieron como modificaciones variables. Las tolerancias de EM/EM fueron de 10 ppm y 0,5 Da, respectivamente. Para identificar los resultados falsos positivos, todas las búsquedas en la base de datos fueron realizados con la opción señuelo habilitada.

\subsubsection{Espectrometría de masas en tándem de alta resolución (LTQ Orbitrap XL)}

Minic et al (2016), describen que los cromopéptidos son separados por HPLC semipreparativa, donde se analizaron mediante espectrometría de masas en tándem de alta resolución utilizando un espectrómetro de masas LTQ Orbitrap XL (Thermo Fisher Scientific Inc., EE. UU.). La ionización se realizó en modo positivo en una sonda de ionización por electrospray calentada (IEC). Los parámetros de IEC fueron: temperatura capilar $275^{\circ} \mathrm{C}$, voltaje de la fuente $4 \mathrm{kV}$, voltaje capilar $5 \mathrm{~V}$, voltaje de la lente del tubo $70 \mathrm{~V}$, flujo de gas auxiliar y de cubierta 12 y 3 (unidades arbitrarias), respectivamente. La adquisición fue de 5 minutos por muestra. Las muestras se inyectaron directamente con un flujo de $10 \mu \mathrm{L} / \mathrm{min}$. Los espectros de EM se adquirieron entre $\mathrm{m} / \mathrm{z} 100 \mathrm{y}$ $\mathrm{m} / \mathrm{z}$ 2000. Los péptidos ionizados se fragmentaron con DIC (disociación inducida por colisión) para obtener espectros de EM2 y EM3. La DIC se realizó con gas helio a una energía de colisión normalizada del $35 \%$ y los iones originales se activaron durante $30 \mathrm{~ms}$. Los datos de ESI-EM, ESI-EM2 y ESI-EM3 se adquirieron con Xcalibur versión 2.1 (Thermo Fisher Scientific Inc., EE. UU.).

\subsection{Identificación de secuencias de FC}

\subsubsection{Identificación de secuencias de FC con Mascot (G. phlegrea)}

Ferraro et al (2020), explican que los espectros de EM/EM adquiridos se transformaron en formato mzData (XML) y se utilizaron para identificación de proteínas con una versión con licencia del software MASCOT (Matrix Science) versión 2.4. con tolerancia EM de 10 ppm y tolerancia EM/EM de 0.6 Da; carga de péptido de (+2) a (+3). Después, se insertó la carboxiamidometalización de la Cys como modificación fija, pero la posible oxidación de las metioninas y la formación de ácido piroglutámico a partir de residuos de glutamina en la posición $\mathrm{N}$ terminal de los péptidos, se consideraron como modificaciones variables para consultar las bases de datos de SwissProt, sin incluir restricciones de la taxonomía.

\subsubsection{Identificación de secuencias de FC con Mascot (Geitlerinema sp)}

De acuerdo con Patel et al (2018), los espectros de huella de masa peptídica (HMP) se analizaron con Mascot (Matrix Science, Reino Unido) para encontrar un parecido en los péptidos con la 
base de datos UniProtKB. El análisis de Mascot se realizó con varios parámetros como la tripsina como enzima, la carbamidometilación (C) como modificación fija, la oxidación (O) como modificación variable, el máximo de escisiones perdidas como 1, la tolerancia de masa del péptido péptido (EM) de $\pm 100 \mathrm{ppm}$ y tolerancia de la masa del fragmento (EM/EM) de $\pm 1 \mathrm{Da}$.

\subsubsection{Identificación de secuencias de FC}

Nair et al (2018), determinaron la secuencia de aminoácidos de las subunidades de R-Ficocianina. El tratamiento con tripsina y análisis posterior de Mascot de las bandas de SDS-PAGE a $18 \mathrm{y}$ $19 \mathrm{kDa}$ respectivamente produjo 6 y 8 péptidos únicos y la digestión de quimotripsina produjo 7 y 15 péptidos. Todos estos espectros de masas de péptidos se comprobaron manualmente para confirmar las secuencias de aminoácidos sugeridas por Mascot. Para mejorar la cobertura de la secuencia e identificar péptidos que no fueron detectados por Mascot, alta calidad de novo. Las etiquetas de secuencia generadas por PEAKS Studio y Novor fueron sometidas a EM-BLAST y verificado manualmente. Analizando cada una de las masas de los espectros de péptidos, la secuencia parcial de la subunidad alfa de ficoeritrina que contiene 104 aminoácidos fue derivado.

De manera similar se determinó la secuencia casi completa de la cadena beta de la ficoeritrina, de $C$. clavulatum, compuesta por 153 residuos de aminoácidos. Finalmente, usando el Programa de búsqueda Blastp, se compararon las secuencias de las ficobiliproteínas derivadas de C. clavulatum con una biblioteca de secuencias de proteínas depositadas en la base de datos SwissProt. La secuencia de la cadena alfa de la ficocianina de $C$. clavulatum mostró un $87 \%$ de identidad con una cadena alfa de Cficocianina aislada de Aglaothamnion neglectum, una macroalga roja marina. Pero según la caracterización espectroscópica, la ficocianina de $C$. clavulatum pertenece a las ficocianinas de tipo $\mathrm{R}$ debido a su característica absorbancia máxima a 551 y $617 \mathrm{~nm}$ y la emisión de fluorescencia máxima a $632 \mathrm{~nm}$. No obstante, la cadena alfa de R-FC de $C$. clavulatum mostró una secuencia del $81 \%$ similitud con la de un alga roja, Porphyridium purpureum.

Del mismo modo, la cadena beta de R-FC de $C$. clavulatum mostró una fuerte similitud de secuencia (85\%) con la de Porphyridium purpureum. La secuencia de aminoácidos de la cadena alfa de ficoeritrina de $C$. clavulatum en comparación con el de las otras algas mostraron identidades de secuencia que van del $44 \%$ al $65 \%$. Pero como se describe, la caracterización espectroscópica de la ficoeritrina aislada de C. clavulatum confirmó explícitamente que pertenece a la R-Ficoeritrina. El análisis de Blastp reveló además que la cadena alfa de R-PE de $C$. clavulatum tiene un $63 \%$ de identidad de secuencia con las proteínas aisladas de dos algas marinas, Griffithsia monilis y Gracilaria chilensis.

\subsubsection{Identificación de secuencias de FC (espectrometría de masas en tándem)}

Minic et al (2016), mencionan que la identificación de los cromopéptidos se realizó mediante la secuenciación manual de novo utilizando el software NIST Mass and Fragment Calculator (Versión: 1.3) para calcular la masa de los fragmentos de una secuencia peptídica de entrada junto con los iones $\mathrm{m} / \mathrm{z}$ correspondientes a los estados de carga (1+), (2+) y (3+).

\section{Caracterización de ficocianina}

\subsection{Purificación}

Ferraro et al (2020), citan que una medida de pureza de las C$\mathrm{FC}$ es la relación A620nm/A280nm (aunque es muy superior a la requerida para aplicaciones alimentarias) y un valor $\geq 4,0$ indica un grado analítico. Los investigadores describen que obtuvieron FCGp mediante un único paso de ultrafiltración recuperando una muestra de proteína con un grado de pureza de 5 y un rendimiento del $80 \%$. Descubrieron que la estructura de la FCGp no está significativamente influenciada por el $\mathrm{pH}$ dentro del rango 4,09,0 , mientras que parece verse afectada por valores altamente ácidos o básicos. Los autores mencionan que la ficocianina sintetizada tiene una alta estabilidad térmica, con valores de temperatura de fusión (Tf) $80{ }^{\circ} \mathrm{C}$ a pH 5,0 y 7,0 (Tabla 1).

La proteína se confirmó además por SDS-PAGE y por espectrometría de masas. Mencionan que como esperaban, la CFGp se caracterizó por presentar dos bandas azules, en línea con otras C-FC, lo que sugiere que está constituida por dos cadenas capaces de unirse al cromóforo. Además, exponen que los pesos moleculares aproximados de las dos subunidades son de 17 y 18 $\mathrm{kDa}$, respectivamente. Aclaran que realizaron pruebas de electroforesis en condiciones no desnaturalizantes (NativePAGE) a $\mathrm{pH} 8,8$ y con resultados, interpretaron que existe una única especie molecular en su muestra. El análisis MASCOT los condujo a la identificación de los péptidos presentes en las secuencias de FCs. No se identificaron proteínas diferentes, confirmando así la pureza de la proteína.

Por otro lado, Patel et al (2018) explican que la extracción de ficocianina se llevó a cabo a partir de un cultivo de Geitlerinema sp. H8DM utilizando el método de lisis mecánica. Describen que la ficocianina registró una pureza de 3,855 y su espectro UV-vis de $\lambda$ max: $620 \mathrm{~nm}$. Ellos resaltan que inicialmente con la prueba de SDS-PAGE se registró una sola banda que indicaba una única subunidad de $18 \mathrm{kDa}$, por otra parte, los datos fueron analizados en una base de datos de proteínas de dominio público (SWISSPROT) donde el peso molecular calculado de las subunidades $\alpha$ y $\beta$ fue de 17,5 $\mathrm{kDa}$ y $18,1 \mathrm{kDa}$ (Tabla 1 ), respectivamente, valores muy similares de peso molecular razón por la cual los autores explican la banda única en SDS-PAGE.

La estabilidad de la FC bajo diferentes condiciones de estrés, como la temperatura, el $\mathrm{pH}$ y diferentes concentraciones del agente oxidante peróxido de hidrógeno $\left(\mathrm{H}_{2} \mathrm{O}_{2}\right)$. Fue muy estable a $4{ }^{\circ} \mathrm{C}$, y al aumentar la temperatura hasta $37{ }^{\circ} \mathrm{C}$ también mostró una buena estabilidad. Sin embargo, aclaran que más allá de esta temperatura, la tasa de degradación es notablemente a $50{ }^{\circ} \mathrm{C}$ y 70 ${ }^{\circ} \mathrm{C}$. Indican que el $\mathrm{pH}$ desempeña un papel muy importante en el mantenimiento de la configuración estructural de las ficobiliproteínas (FBP) y a partir de esto, demuestran que la estabilidad de la ficocianina a pH 4 y 7 se encontró muy alta. No obstante, a pH 9 y pH 11, visualizaron que el plegado de la proteína se modificó ligeramente, pero bajo un $\mathrm{pH}$ extremadamente ácido de 2 la estabilidad de la FC se modificó drásticamente. Como mencionaron, se realizó la incubación de la FC purificada con el agente oxidante $\mathrm{H}_{2} \mathrm{O}_{2}$ y esto condujo a una sucesiva disminución de su absorción con el aumento de la concentración del reactivo, acompañada de la desaparición de su respectivo color. Añaden que, a diferencia de la alta temperatura y el cambio de $\mathrm{pH}$, las propiedades espectrales de la FC se mantuvieron bajo el estrés oxidativo, lo que indica su potencial como captadores de radicales libres.

Nair et al (2018), explican que el proceso de extracción se mantuvo a $4{ }^{\circ} \mathrm{C}$. Los extractos crudos de algas se precipitaron utilizando soluciones saturadas de sulfato de amonio y observaron que las obtenidas entre el 30 y el 50\% de saturación tenían colores brillantes que indican la presencia de ficobiliproteínas. Los 
autores determinaron las concentraciones de proteínas en las fracciones dializadas y mencionan que en la fracción obtenida tras la saturación del $40 \%$ de $\left(\mathrm{NH}_{4}\right)_{2} \mathrm{SO}_{4}$ registró la máxima concentración de proteínas y fue posteriormente seleccionada para su purificación y caracterización. El precipitado seleccionado se aplicó a una columna de intercambio con un gradiente lineal de $\mathrm{NaCl}$ preparado en tampón de fosfato sódico 0,05 M (pH 7.4). Nair et al (2018), describen que el cromatograma mostró una separación exitosa del constituyente de las ficobiliproteínas con dos picos preponderantes. El eluido correspondiente al pico 1 tenía un color azul intenso que se eluyó con $\mathrm{NaCl} 0,4 \mathrm{M}$ y sospechaban que esta fracción contenía ficocianina debido a su característico color azul. El segundo pico que se eluyó con una concentración de $0,7 \mathrm{M}$ de $\mathrm{NaCl}$ se especuló que era ficoeritrina debido a su color rosa intenso.

Mencionan que la primera fracción (parte importante dentro de este estudio) mostró máximos de absorción a $551 \mathrm{~nm}$ y $617 \mathrm{~nm}$ indicativos de la presencia de R-Ficocianina (R-FC). El pico característico a $617 \mathrm{~nm}$ se genera, según se informan, a partir de la asociación del cromóforo de la ficocianobilina con la proteína. El índice de pureza de la fracción R-FC resultó ser de 5,01 (Tabla 1). Ellos explican que estos valores son relativamente superiores a 4,5, una medida que ayuda a considerar las ficobiliproteínas para sus aplicaciones farmacéuticas y fluorescentes.

Minic et al (2016), realizaron la digestión de C-FC mediante SDS-PAGE, tras la digestión con pepsina en condiciones gástricas simuladas, sus resultados demuestran que la pepsina libera rápidamente (después de 0,5 minutos) pequeños péptidos, visibles como manchas en el fondo del gel de separación que disminuyen gradualmente con el aumento del tiempo de incubación de la digestión. Según con lo anterior, los investigadores manifiestan que esto sugiere que la C-FC es muy susceptible a la digestión con pepsina en las condiciones aplicadas, y está de acuerdo con la inestabilidad de la C-FC por debajo de $\mathrm{pH} 4$ (Tabla 1); resaltan que después de desplegarse a $\mathrm{pH}$ ácido, la proteína se vuelve totalmente accesible a la enzima. Registraron las marcas de las subunidades $\alpha(17 \mathrm{kDa})$ y $\beta(19 \mathrm{kDa})$ de la C-FC.

Posteriormente, exponen que los péptidos liberados se separaron mediante HPLC de los cuales se aislaron cinco fracciones dominantes de péptidos que contenían el cromóforo FCB (I-V). Explican que hay una buena concordancia entre las formas de los picos a $615 \mathrm{~nm}$ (absorción del cromóforo), 280 (absorción del cromóforo y de los residuos de aminoácidos aromáticos) y $215 \mathrm{~nm}$ (absorción del cromóforo y de los enlaces peptídicos). La cromatografía de las fracciones que aislaron ha mostrado que la pureza relativa de los cromopéptidos (según la absorbancia a $215 \mathrm{~nm}$ ) en las fracciones II-V estaba entre el 91 y el $98 \%$, mientras que el cromopéptido de la fracción I tenía una pureza del $72 \%$. Por lo tanto, se puede concluir que los cromopéptidos obtenidos después de la HPLC tienen un mayor grado de pureza.

Tabla 1: Estabilidad de ficocianinas

\begin{tabular}{|c|c|c|c|c|c|}
\hline Artículo & Cianobacteria & $\begin{array}{c}\mathrm{T} \\
\left({ }^{\circ} \mathrm{C}\right)\end{array}$ & $\mathrm{pH}$ & $\begin{array}{c}\text { Peso } \\
\text { molecular } \\
(\mathrm{kDa})\end{array}$ & $\begin{array}{c}\text { Pureza } \\
\text { total }\end{array}$ \\
\hline $\begin{array}{c}\text { Ferraro et } \\
\text { al (2020) }\end{array}$ & G. phlegrea & 80 & $5-7$ & $\begin{array}{c}\alpha 17 \\
\beta 18\end{array}$ & 5 \\
\hline $\begin{array}{c}\text { Patel et al } \\
(2018)\end{array}$ & $\begin{array}{c}\text { Geitlerinema } \\
\text { sp. }\end{array}$ & $4-37$ & $4-7$ & $\begin{array}{c}\alpha 17 \\
\beta 18\end{array}$ & 3,855 \\
\hline $\begin{array}{c}\text { Nair et al } \\
\text { (2018) }\end{array}$ & $\begin{array}{c}C . \\
\text { clavulatum }\end{array}$ & 4 & 7,4 & $\begin{array}{c}\alpha 17,5 \\
\beta 18,1\end{array}$ & 5,01 \\
\hline $\begin{array}{c}\text { Minic et } \\
\text { al (2016) }\end{array}$ & Spirulina & 37 & 4 & $\begin{array}{c}\alpha 17 \\
\beta 21\end{array}$ & 8,7 \\
\hline
\end{tabular}

\subsection{Análisis de proteínas}

Ferraro et al (2020) explican que, las dos bandas correspondientes a las cadenas $\beta$ y $\alpha$ se extrajeron del gel y se sometieron a hidrólisis con tripsina y quimotripsina para garantizar la mayor cobertura posible de la secuencia de las dos cadenas. Posteriormente, las mezclas de péptidos que se obtuvieron se analizaron mediante CLEM/EM; para la identificación de las proteínas utilizaron el software interno MASCOT (Matrix Science). Ellos reportan las secuencias recogidas a partir de los espectros de fragmentación $\mathrm{EM} / \mathrm{EM}$ relacionados con la cadena $\alpha$ tras el tratamiento y las puntuaciones asociadas a cada una de ellas. Hicieron análisis similares en las bandas de proteínas correspondientes a la cadena $\beta$. Sobre la base de datos de la secuenciación espectral de masas basados en la proteólisis y tomando en cuenta la alta identidad de secuencia entre las C-ficocianinas de diferentes fuentes, se determinaron las secuencias de las cadenas $\alpha$ y $\beta$ de FCGp. Patel et al (2018), mencionan que el análisis de dos manchas obtenidas por el gel 2D se exploró mediante MALDI-TOF-EM/EM. El espectro de una mancha digerida por tripsina a $\sim 5 \mathrm{pI}$ mostró cinco péptidos principales 898, 998, 1455, 1744 y 1760 Da de la subunidad $\alpha$ (Fig. 2), mientras que, la mancha a $\sim 4$ pI mostró 859 , 1404, 1877, 2393 y 2410 Da de la subunidad $\beta$ (Fig. 3), explican que las secuencias de aminoácidos de estos péptidos mostraron un alto grado $(100 \%)$ de similitudes con la secuencia de nucleótidos de la FC $\alpha$-subunidad (Accession\#LT671830) y de la FC $\beta$ subunidad (Accession\#LT671831) con una cobertura de secuencia del $29 \%$ y $34 \%$, respectivamente.

Nair et al (2018), describen que con la ayuda del software Mascot identificaron de la primera banda $(17 \mathrm{kDa}) 15$ péptidos y de la segunda $(21 \mathrm{kDa})$ registraron 8 péptidos. Ellos expresan que estos péptidos mostraron similitud de secuencia con las subunidades alfa y beta de la R-ficocianina de Vertebrata lanosa, un alga roja marina.

Minic et al (2016), menciona que los cromopéptidos separados por HPLC se secuenciaron mediante espectrometría de masas en tándem. Las secuencias de los cromopéptidos se determinaron a partir de los espectros de EM de las fracciones de cromopéptidos (Fig. 4), utilizando sus masas moleculares calculadas, las secuencias conocidas de las subunidades $\alpha$ (UniProtKB-P72509) y $\beta$ (UniProtKB-P72508) de C-FC y la posición de unión de FCB. La confirmación de las secuencias se realizó mediante el análisis de los espectros EM2 (Fig. 5) y EM3 (Fig. 6) de los iones madre, y los espectros EM y EM2 del FCB puro. El espectro EM del FCB muestra un ion dominante característico a m/z 587,29; en el espectro EM2 del FCB los iones más dominantes con relaciones m/z 464,22 y 299,14: el espectro EM3 del ion con m/z 299,14 contiene el ion con $\mathrm{m} / \mathrm{z} 271,14$. Explican que al igual que en otras bilinas, el ion con relación $\mathrm{m} / \mathrm{z}$ 464,22 es el resultado de la pérdida de los dos anillos de pirrol terminales, mientras que el ion con $\mathrm{m} / \mathrm{z}$ 299,14 es el resultado de la ruptura del enlace C-C entre el carbono de metileno del puente central a cualquiera de los dos anillos de pirrol interiores en la molécula de FCB. El ion adicional con relación $\mathrm{m} / \mathrm{z} 271,14(\mathrm{z}=1)$ corresponde a la pérdida natural de $\mathrm{CO}$ del ion producto a $\mathrm{m} / \mathrm{z} 299,14$. El CID escinde el enlace tioéter entre el cromóforo del FCB y el péptido, por lo que los iones que representan al FCB y a los péptidos sin cromóforo aparecen en los espectros EM2 de los cromopéptidos.

La fragmentación posterior del ion con una relación $\mathrm{m} / \mathrm{z}$ de 587,29 da lugar a iones con una relación $\mathrm{m} / \mathrm{z}$ de 464,22, 299,14 y 271,14 en el espectro EM3 apropiado. Estos iones fueron la confirmación de que el ion padre es el cromopéptido y sólo se analizaron los iones cuya fragmentación da lugar a iones derivados del cromóforo. La fragmentación de los iones del péptido sin cromóforo en el espectro EM2 da un espectro EM3 
con series de iones, lo que confirma las secuencias del cromopéptido. En general, mencionan que los péptidos bioactivos suelen contener entre 3 y 20 residuos de aminoácidos, por lo que el pequeño tamaño de los cromopéptidos obtenidos indica su potencial bioactividad.

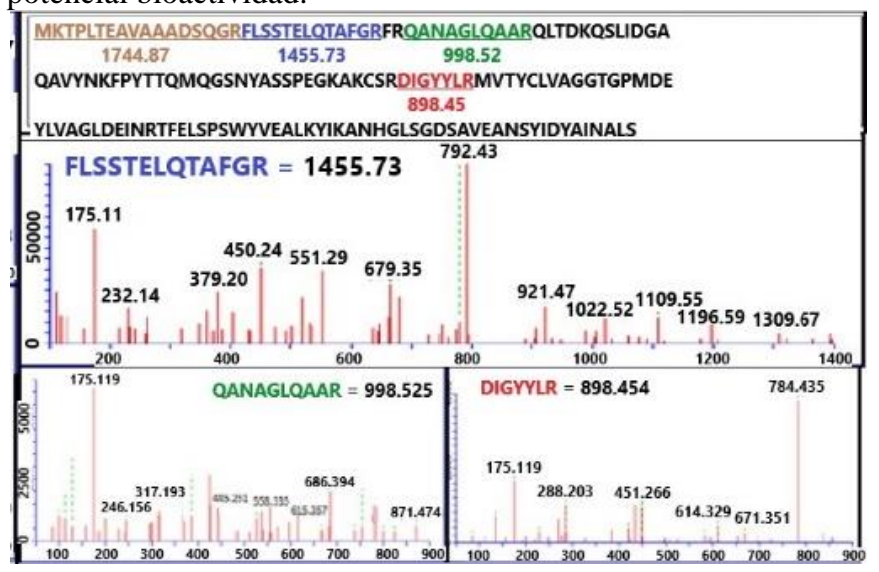

Figura 2. Espectro de cadena $\alpha$ por MALDI-TOF-EM / EM (Patel et al, 2018).

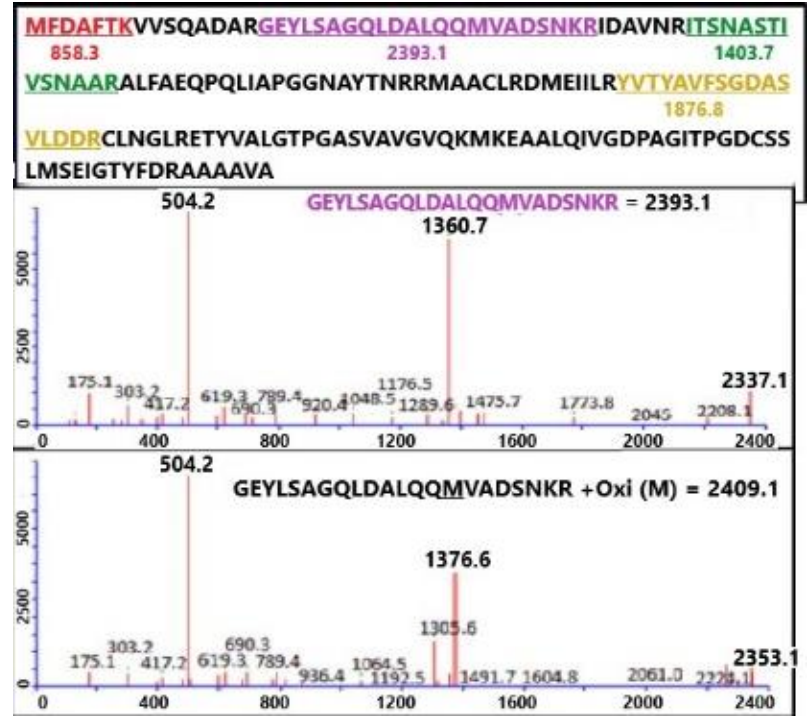

Figura 3. Espectro de cadena $\beta$ por MALDI-TOF-EM/EM (Patel et al, 2018).

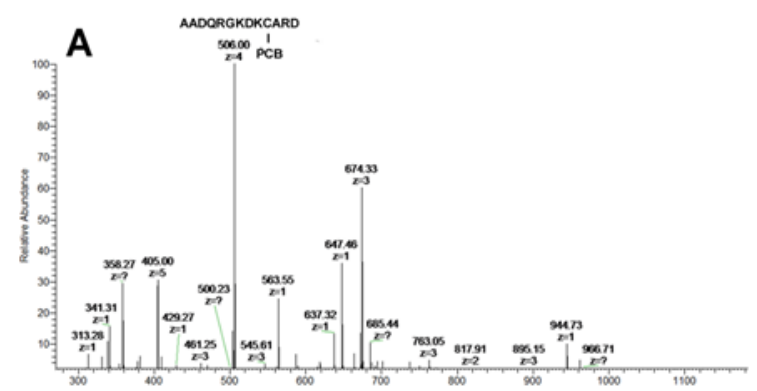

Figura 4. Espectro EM de la fracción I, tras 24 h de digestión de C-PC por pepsina (Minic et al, 2016).

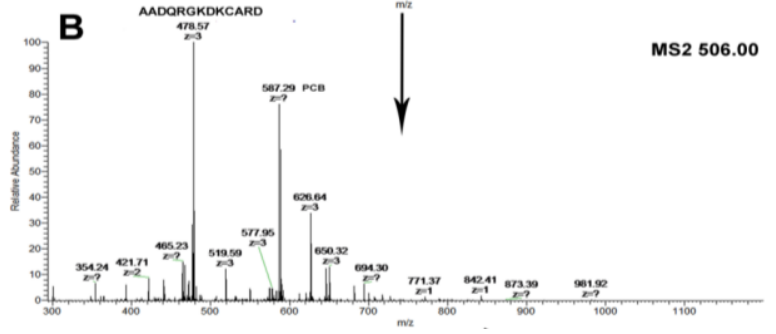

Figura 5. Espectro EM2 de ion molecular con relación $\mathbf{m} / \mathbf{z}$ $506,00(z=4)$ (Minic et al, 2016).

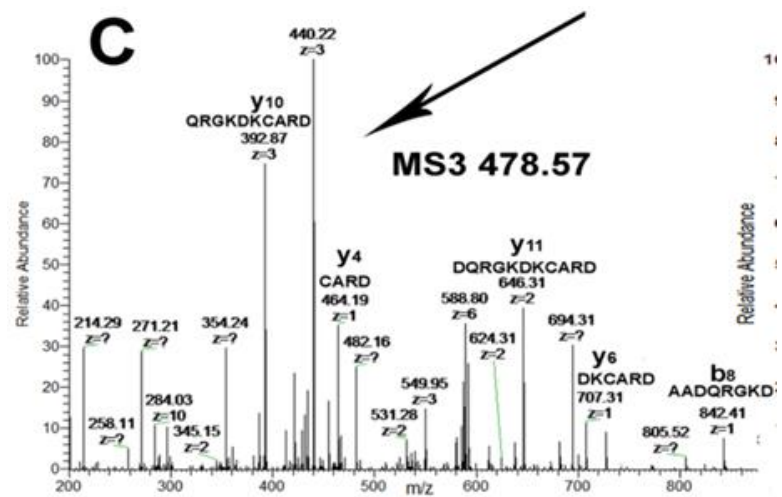

Figura 6. Fragmentación del ion con relación $\mathrm{m} / \mathrm{z}$ 478,57 $(\mathrm{z}=3)$ (Minic et al, 2016).

\section{Conclusiones}

Las ficocianinas han atraído el interés de la comunidad científica por varias razones. Pueden utilizarse como colorantes alimentarios y son capaces de ejercer numerosas actividades biológicas de interés para la investigación farmacéutica y biomédica. De acuerdo con los artículos científicos recopilados, se determinó que el pH óptimo de la ficocianina es de 4-9 y los autores trabajaron con diferentes temperaturas, pero no mayores a $30{ }^{\circ} \mathrm{C}$, ya que describieron que la inestabilidad de la ficobiliproteína comenzaba a los $50{ }^{\circ} \mathrm{C}$ aproximadamente.

De acuerdo con los resultados obtenidos por espectrofotometría de masas los autores detectaron las dos subunidades características de la ficocianina $(\alpha$ y $\beta)$ con pesos moleculares de $17 \mathrm{kDa}$ y $18 \mathrm{kDa}$, respectivamente; además, presentaron la secuencia de la proteína con ayuda del software MASCOT que fue uno de los más empleados en los artículos revisados.

\section{Agradecimientos}

Los autores agradecen el apoyo por uso de instalaciones al centro de cooperación academia industria de la Universidad Tecnológica de Tecámac.

\section{Referencias}

Busch, K. L. (Third Edition)., (2003). Encyclopedia of Physical Science and Technology. Elsevier.

Chen, H. W., Yang, T. S., Chen, M. J., Chang, Y.C., Wang E. I., Ho, C. L. Lai, Y. J., Yu, C. C., Chou, J. C., Ping Chao, L. K., Liao, P. C., (2014) Purification and immunomodulating activity of C-phycocyanin from Spirulina platensis cultured using power plant flue gas. Process Biochem 49, 1337- 1344 .

DOI: 10.1016/j.procbio.2014.05.006 
Ferraro, G., Imbimbo, P., Marseglia, A., Illiano, A., Fontanarosa, C., Amoresano, A., Olivieri, G., Pollio, A., Monti, D.M., Merlino, A., (2020). A thermophilic C-phycocyanin with unprecedented biophysical and biochemical properties. International Journal of Biological Macromolecules 150, 38-51.

DOI: 10.1016/j.ijbiomac.2020.02.045

Fratelli, C., Burck, M., Amarante, M.C.A., Braga, A.R.C., (2021). Antioxidant potential of nature's "something blue": Something new in the marriage of biological activity and extraction methods applied to C-phycocyanin. Trends in Food Science \& Technology 107, 309-323. DOI: $10.1016 /$ j.tifs.2020.10.043

Grover, P., Bhatnagar, A., Kumari, N., Bhatt, A. N., Nishad, D. K., Purkayastha, J., (2021). C-Phycocyanin-a novel protein from Spirulina platensis- In vivo toxicity, antioxidant and immunomodulatory studies. Saudi Journal of Biological Sciences 28, 1853-1859. DOI: 10.1016/j.sjbs.2020.12.037

Hoffmann, E., Stroobant, V. (Third Ed.), (2007). Mass Spectrometry: Principles and Applications. Wiley. Brussels, Belgium.

Hsieh Lo, M., Castillo, G., Ochoa Becerra, M. A., Mojica, L., (2019). Phycocyanin and phycoerythrin: Strategies to improve production yield and chemical stability. Algal Research 42, 101600. DOI: $10.1016 /$ j.algal.2019.101600

Ismaiel, M. M. S., El-Ayouty, Y. M., Piercey Normore, M. D., (2016). Role of $\mathrm{pH}$ on antioxidants production by Spirulina (Arthrospira) platensis. Brazilian Journal of Microbiology 47, 298-304. DOI: 10.1016/j.bjm.2016.01.003

Kaldmäe, M., Sahin, C., Saluri, M., Marklund, Erik G., Landreh, M., (2019). A strategy for the identification of protein architectures directly from ion mobility mass spectrometry data reveals stabilizing subunit interactions in light harvesting complexes. The Protein Society 28, 1024-1030. DOI: $10.1002 /$ pro.3609

Koníčková, R., Vaňková, K., Vaňková, J., Váňová, K., Muchová. L., Subhanová, I., Zadinová, M., Zelenka, J., Dvořák, A., Kolář, M., Strnad, H., Rimpelová, S., Ruml, T., Wong, R. J., Vítek, L., (2014). Anti-cancer effects of blue-green alga Spirulina platensis, a natural source of bilirubinlike tetrapyrrolic compounds. Annals of Hepatology 13, 273-283. DOI: $10.1016 / \mathrm{S} 1665-2681(19) 30891-9$

Milman, B. L., (2015). General principles of identification by mass spectrometry. TrAC Trends in Analytical Chemistry 69, 24-33.
DOI: 10.1016/j.trac.2014.12.009

Minic, S. L., Stanic-Vucinic, D., Mihailovic, J., Krstic, M., Nikolic, M. R., Velickovic, T. C., (2016). Digestion by pepsin releases biologically active chromopeptides from C-phycocyanin, a blue-colored biliprotein of microalga Spirulina. Journal of Proteomics 147, 132-139. DOI 10.1016/j.jprot.2016.03.043

Nair, D., Krishna, J. G., Panikkar, M.V.N., Nair, B.G., Pai, J.G., Nair, S.S., (2018). Identification, purification, biochemical and mass spectrometric characterization of novel phycobiliproteins from a marine red alga, Centroceras clavulatum. International Journal of Biological Macromolecules 114, 679-691.

DOI: 10.1016/j.ijbiomac.2018.03.153

Patel, H. M., Rastogi, R. P., Trivedi, U., Madamwar, D., (2018). Structural characterization and antioxidant potential of phycocyanin from the cyanobacterium Geitlerinema sp. H8DM. Algal Research 32, 372-383. DOI: 10.1016/j.algal.2018.04.024

Prabakaran, G., Sampathkumar, P., Kavisri, M., Moovendhan, M., (2020). Extraction and characterization of phycocyanin from Spirulina platensis and evaluation of its anticancer, antidiabetic and antiinflammatory effect. International Journal of Biological Macromolecules 153, 256-263. DOI: 10.1016/j.ijbiomac.2020.03.009

Raj, T. K., Ranjithkumar, R., Kanthesh, B. M., Gopenath, T. S., (2020). Cphycocyanin of spirulina plantesis inhibits NSP12 required for replication of SARS-COV-2: A novel finding in-silico. International Journal of Pharmaceutical Sciences and Research 11, 4271-4278. DOI: 10.13040/IJPSR.0975-8232.11(9).4271-78

Ratha, S. K., Renuka, N., Rawat, I., Bux, F., (2021). Prospective options of algae-derived nutraceuticals as supplements to combat COVID-19 and human coronavirus diseases. Nutrition 83, 111089. DOI: $10.1016 /$ j.nut.2020.111089

Rivera, C., Niño, L., Gelves, G., (2021). Modeling of phycocyanin production from Spirulina platensis using different light-emitting diodes. South African Journal of Chemical Engineering 37, 167-178. DOI: 10.1016/j.algal.2018.04.024

Van Bergen, W., Heck, A. J. R., Baggelaar, M. P., (2021). Recent advancements in mass spectrometry-based tools to investigate newly synthesized proteins. Current Opinion in Chemical Biology. DOI: 10.1016/j.cbpa.2021.07.001 